\title{
ORIGINAL ARTICLE Differential effects on gene transcription and hematopoietic differentiation correlate with GATA2 mutant disease phenotypes
}

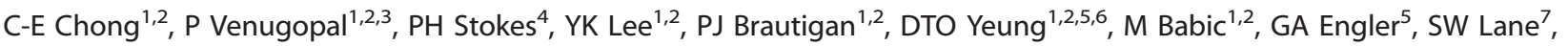 \\ M Klingler-Hoffmann ${ }^{3}$, JM Matthews ${ }^{4}$, RJ D'Andrea ${ }^{2,5,8}$, AL Brown ${ }^{1,2}$, CN Hahn ${ }^{1,2,6}$ and HS Scott ${ }^{1,2,3,6,8,9}$
}

Heterozygous GATA2 mutations underlie an array of complex hematopoietic and lymphatic diseases. Analysis of the literature reporting three recurrent GATA2 germline (g) mutations (gT354M, gR396Q and gR398W) revealed different phenotype tendencies. Although all three mutants differentially predispose to myeloid malignancies, there was no difference in leukemia-free survival for GATA2 patients. Despite intense interest, the molecular pathogenesis of GATA2 mutation is poorly understood. We functionally characterized a GATA2 mutant allelic series representing major disease phenotypes caused by germline and somatic (s) mutations in zinc finger 2 (ZF2). All GATA2 mutants, except for sL359V, displayed reduced DNA-binding affinity and transactivation compared with wild type (WT), which could be attributed to mutations of arginines critical for DNA binding or amino acids required for ZF2 domain structural integrity. Two GATA2 mutants (gT354M and gC373R) bound the key hematopoietic differentiation factor PU.1 more strongly than WT potentially perturbing differentiation via sequestration of PU.1. Unlike WT, all mutants failed to suppress colony formation and some mutants skewed cell fate to granulocytes, consistent with the monocytopenia phenotype seen in GATA2-related immunodeficiency disorders. These findings implicate perturbations of GATA2 function shaping the course of development of myeloid malignancy subtypes and strengthen complete or nearly complete haploinsufficiency for predisposition to lymphedema.

Leukemia (2018) 32, 194-202; doi:10.1038/leu.2017.196

\section{INTRODUCTION}

Germline predisposition to hematological malignancies is an increasingly recognized clinical entity. ${ }^{1}$ More than 12 genes with germline mutations leading to autosomal dominant inheritance of hematological malignancies have been described, with several genes, RUNX1, ${ }^{2}$ CEBPA, ${ }^{3}$ GATA2, ${ }^{4}$ ANKRD $26^{5}$ and DDX $41,{ }^{6}$ predipsosing to myelodysplastic syndrome and/or acute myeloid leukemia (MDS/AML). With notable exceptions (for example, DDX41), individuals with a family history of hematological malignancies generally present at earlier ages of diagnosis compared to sporadic disease; for example, GATA2 germline (g) mutations transmitted as autosomal dominant predisposition to early onset MDS/AML with often aggressive disease course and poor outcome. ${ }^{4}$ Consistent with this, germline GATA2 mutations were recently reported in pediatric MDS, where they were often de novo germline events. ${ }^{7}$ Patients harboring germline GATA2 mutations can manifest an array of complex diseases with overlapping phenotypes including Emberger syndrome (primary lymphedema with $\mathrm{MDS} / \mathrm{AML})^{8-10}$ and immunodeficiency (ID) disorders such as monocytopenia and mycobacterial infection (MonoMac) syndrome, ${ }^{11,12}$ dendritic cell, monocyte, B and NK lymphoid (DCML) deficiency syndrome, ${ }^{13,14}$ chronic neutropenia, ${ }^{15}$ natural killer cell deficiency ${ }^{16}$ and aplastic anemia, ${ }^{17,18}$ all of which have an increased propensity to develop MDS/AML. These mutants often possess compromised transcriptional activity and have dominant-negative characteristics in some settings. Patients with complete or partial gene deletions may develop Emberger syndrome, indicating that complete GATA2 haploinsufficiency may be required for predisposition to lymphedema. $8,10,19$

Somatic GATA2 mutations are found in specific sporadic myeloid leukemia subtypes, that is, chronic myeloid leukemia in blast crisis, $^{20}$ monocytic AML (FAB M5) ${ }^{21}$ and cytogenetically normal AML with mono- or biallelic CEBPA mutations. ${ }^{22-25}$ Somatic missense mutations occur predominantly in zinc finger 1 (ZF1) of GATA2, with the exception of $s L 359 V^{20}$ and $s R 362 Q^{22-25}$ in ZF2. In contrast, no missense germline mutations occur within ZF1, rather all occur in the ZF2 domain and the neigboring C-terminal region. ${ }^{4,11,12}$ Phenotypic variation and unique mutational clustering separating germline and somatic GATA2 mutations imply that they represent at least two separate entities. In this study, we systematically analyzed genetic and clinical data of patients carrying three most common germline GATA2 ZF2 mutations (gT354M, gR396Q and gR398W), which revealed that although these mutations broadly predispose to $M D S / A M L$, they appear to

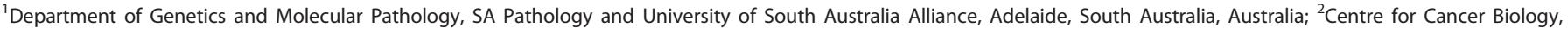

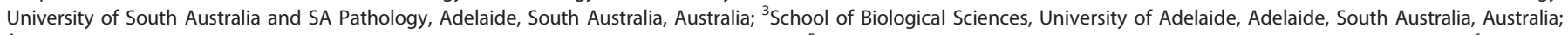

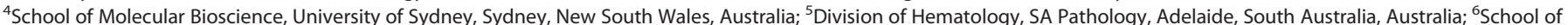

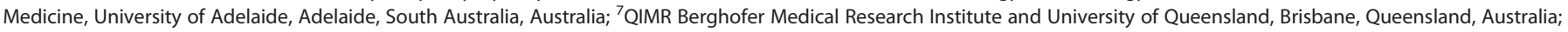

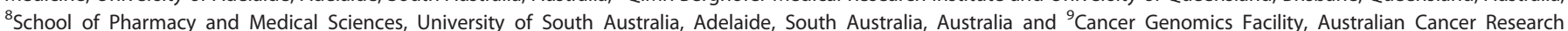

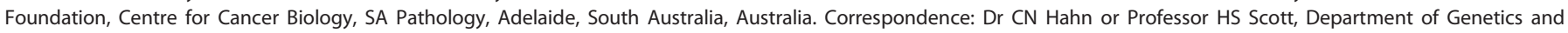
Molecular Pathology, Centre for Cancer Biology, SA Pathology and University of South Australia Alliance, Frome Road, Adelaide, SA 5000, Australia. 


\begin{tabular}{|c|c|c|c|c|c|c|}
\hline Mutation $(\mathrm{n}=98)$ & $\begin{array}{l}\text { Myeloid malignancies } \\
\text { (MDS/AML/aCML/CMML) }\end{array}$ & $\begin{array}{l}\text { ID with myeloid } \\
\text { malignancies }\end{array}$ & $\begin{array}{c}\text { ID without myeloid } \\
\text { malignancies }\end{array}$ & ES (lymphedema+MDS) & No symptoms & Total \\
\hline $\operatorname{gT354M}(n=64)$ & 39 (64\%) & $8(13 \%)$ & $4(7 \%)$ & 0 & $10(16 \%)$ & $61^{a}$ \\
\hline gR396Q $(n=19)$ & $6(33 \%)$ & $10(55 \%)$ & $1(6 \%)$ & $1(6 \%)$ & 0 & $18^{\mathrm{a}}$ \\
\hline
\end{tabular}

confer unique characteristics leading to different disease subtypes and age of onset. Using a GATA2 ZF2-mutant allelic series representing major hematological disease subtypes, we show differential impact on GATA2 DNA-binding affinity, transactivation, colony formation, differentiation, protein-protein interactions and/or ZF2 structural integrity. We also report a genotypephenotype correlation where GATA2 mutants with residual activity render resistance to lymphedema, while predisposing to myeloid malignancy and/or ID, and where specific ZF2 mutations display a propensity for particular myeloid malignancy subtypes.

\section{MATERIALS AND METHODS}

Patient information

Genetic and clinical information correlated with common germline GATA2 mutations (gT354M, gR396Q and gR398W) was available from 98 patients, $4,7,8,11-16,19,26-33$ gathered in studies from 27 confirmed kindreds and 7 individuals with de novo mutations (Supplementary Table 1). All data were collected with informed consent and approval from Institutional Review Board/Human Research Ethics Committees.

\section{Constructs}

GATA2 expression constructs (human (h) pCMV6-XL6-GATA2 and mouse $(m)$ pCMV6-Entry-GATA2) were purchased from OriGene (Rockville, MD, USA). A GATA2 mutant allelic series of expression constructs (human and mouse) were generated by site-directed mutagenesis (QuickChange II, Stratagene, La Jolla, CA, USA). FLAG-tagged hGATA2 (wild type (WT) and mutants) was subcloned into the pcDNA3. A DNA fragment corresponding to the hGATA2 CDNA ZF2 domain (WT and mutants, residues 328-409) was sub-cloned into the pET11a (Novagen, San Diego, CA, USA) and proteins were expressed in bacteria, purified and characterized as described. ${ }^{34}$ For retroviral expression constructs, mGata2 WT and mutant cDNA coding regions were sub-cloned into the pMSCV-IRES-GFP (Supplementary Table 2).

\section{Cell isolation and transplantation assays}

Female C57BL/6 mice $\mathrm{Lin}^{-} \mathrm{Sca}^{+} \mathrm{c}^{+} \mathrm{Kit}^{+}$(LSK) cells were transduced with pMSCV-mGata2-IRES-GFP (see Supplementary Information). For transplantation assays, $1 \times 10^{5} \mathrm{GFP}^{+}$LSK cells and $2 \times 10^{5}$ helper bone marrow cells were injected into the tail vein of lethally irradiated (1000 Rad) congenic mice. All animal studies were approved by the University of Adelaide and SA Pathology Animal Ethics Committees.

\section{Statistical analysis}

The Mann-Whitney test and Fisher's exact test (two-tailed test) were used to analyze statistical significance of observed differences. Survival and time to event analyses were performed using Kaplan-Meier, and Fine and Gray $^{35}$ methods, respectively, in R.

\section{RESULTS}

Recurrent germline GATA2 mutations are associated with different clinical manifestation

To understand the clinical outcomes of the most common germline GATA2 mutations, a review of in-house clinical information and the literature was undertaken (Supplementary Table 1). Survival and time to event (development of hematological and/or immunological disorder) data for 94 patients with the three most common mutations (gT354M, gR396Q and gR398W) was calculated (Table 1). Common to all mutations was the development of MDS/AML with moderate-high penetrance (75/94, 80\%). However, age of MDS/AML onset was different among patients with different GATA2 mutations ( $P=0.04)$; gR396Q patients being the earliest to develop an event (median age 16) compared with gT354M and gR398W patients (median age 27 and 39 years, respectively) (Figure 1a). Patients with gR398W usually presented with an ID at around the same time as the initial diagnosis of the myeloid disorder (median age 35 years) as did about $67 \%$ of the gR396Q patients (Figure 1b). In contrast, gT354M patients usually presented with MDS/AML as the first sign of the clinical syndrome, which may mask or confound the diagnosis of immune defects (Figures $1 \mathrm{~b}$ and $\mathrm{c}$ ). Overall, the leukemia-free survival of the three mutations was equally poor (Figure 1d). Interestingly, of 50 individuals with gT354M-driven myeloid malignancies, there were 30 AML (60\%) and no chronic myelomonocytic leukemia cases, whereas for 12 affected with gR398W there were only 2 cases associated with disease progression to AML (17\%), but 4 chronic myelomonocytic leukemia (33\%) (Table 2). No chronic myelomonocytic leukemia was seen in the $18 \mathrm{gR3960}$ cases, although lymphedema was reported in 1 individual. ${ }^{30}$ Collectively, this data demonstrate prognostically important mutation-specific differences in disease subtype, penetrance and outcome.

GATA2 mutants display differentially altered DNA binding via different mechanisms

To investigate the functional basis of differences in GATA2-driven disease, we generated a mutant allelic series for human GATA2 ( $h$ GATA) representing major disease phenotypes: MDS/AML (gT354M and gT355del), ${ }^{4}$ chronic myeloid leukemia in blast crisis (sL359V), ${ }^{20}$ Emberger syndrome (gR361L and gC373R), ${ }^{10}$ acute monocytic leukemia (AML-M5), ${ }^{21}$ biallelic CEBPA AML $(\mathrm{sR362Q})^{22,23,36,37}$ and MonoMAC/DCML deficiency syndrome (ID with MDS/AML, gR398W) ${ }^{11-14}$ (Figure 2a). Previously reported mutants gT354M, gT355del and SL359V were included for the purposes of comparison. ${ }^{4}$ We also made corresponding mutant murine GATA2 clones (mGATA2). In HEK293 cells, all mutant proteins displayed nuclear localization similar to WT GATA2 (Supplementary Figure S1), indicating that, unlike RUNX1, disruption of the nuclear localization signal is not a major mechanism for GATA2 functional dysregulation. ${ }^{4}$ Examination of DNA binding to 


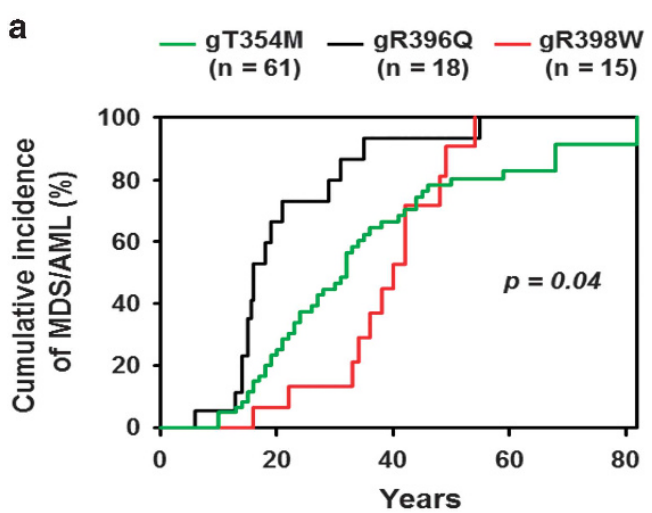

C

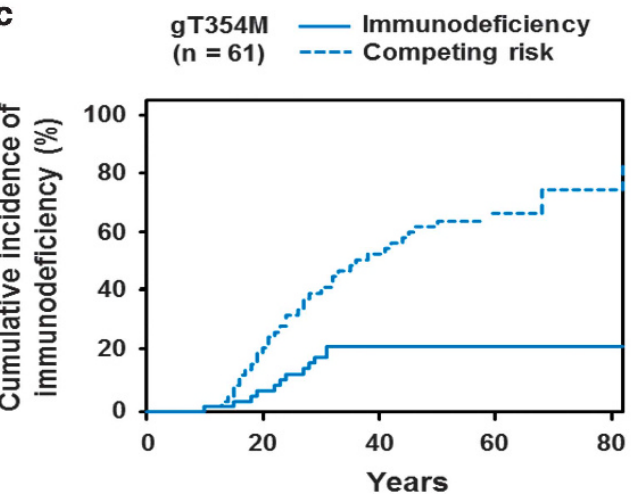

b
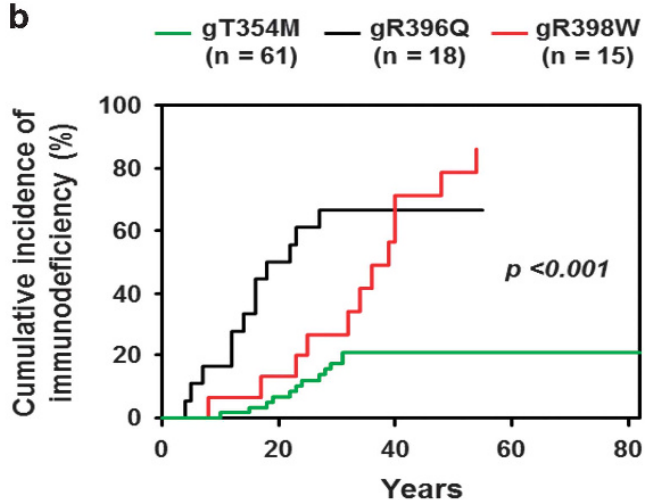

d
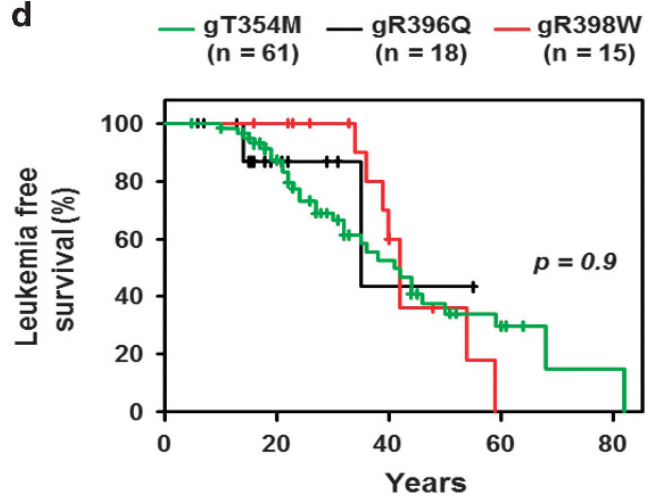

Figure 1. Time to event analyses were performed using the cumulative incidence function with adjustment for competing risk. (a) Cumulative incidence of MDS/AML stratified by GATA2 mutations. (b) Cumulative incidence of ID for three germline GATA2 mutations. (c) Cumulative incidence of ID syndrome in gT354M patients. AML, MDS and death from any cause were considered competing risk events that prevent or mask the development of ID. (d) Kaplan-Meier plot of leukemia transformation free survival for GATA2 mutants. An event is either diagnosis of AML or death. All $P$-values (log-rank test) are for overall global comparisons.

Table 2. Recurrent germline GATA2 ZF2 mutations and their association with myeloid malignancy subtypes

\begin{tabular}{llcccc}
\hline Mutation & \multicolumn{5}{c}{ Myeloid malignancy subtypes } \\
\cline { 2 - 6 } & aCML/CMML & MDS & AML & Total $^{\text {a }}$ & P-value $^{\text {b }}$ \\
\hline gT354M & $1(2 \%)^{\mathrm{c}}$ & $19(38 \%)$ & $\mathbf{3 0}(\mathbf{6 0 \% )}$ & 50 & \\
gR396Q & 0 & $\mathbf{1 4}(\mathbf{7 8} \%)$ & $4(22 \%)$ & 18 & $<0.001$ \\
gR398W & $4(33 \%)^{\mathrm{d}}$ & $\mathbf{6 ( 5 0 \% )}$ & $2(17 \%)$ & 12 & \\
& & & & 80 &
\end{tabular}

Abbreviations: $\mathrm{AML}$, acute myeloid leukemia; $\mathrm{aCML}$, atypical chronic myeloid leukemia; CMML, chronic myelomonocytic leukemia; MDS, myelodysplastic syndrome. ${ }^{\text {a Unaffected individuals }(n=11) \text { or individuals }}$ with ID syndrome alone $(n=7)$ were excluded from statistical analysis. $\mathrm{b}_{3} \times 3$ Fisher's exact probability test (two-tailed test) for GATA2 mutants, aCML/CMML, MDS and AML. ${ }^{\mathrm{C}} \mathrm{CCML}$. ${ }^{\mathrm{d}} \mathrm{CMML}$ (unique for gR398W). Predominant phenotype (bold).

known GATA2-responsive elements using western blotting electromobility shift assays revealed a single GATA2-DNA complex for WT GATA2 (Figures $2 b$ and $c$ ). mGATA2 mutant proteins and $h$ GATA2 (Supplementary Figures S2 and S3) exhibited very similar DNA-binding patterns for both hGM-CSF and hTCRD probes. A range of relative DNA-binding capabilities were identified that could be divided into three classes. SL359V displayed no significant reduction in binding (Class I) in contrast to gT354M, sR362Q, gR398W and sL321F (ZF1 mutant), which had reduced but detectable binding (Class II), whereas gT355del,
gR361L, gC373R and gR396Q (Class III) displayed little or no detectable binding (Figure $2 \mathrm{c}$ and Supplementary Figures S2). DNA binding was confirmed and quantified using isothermal titration calorimetry (Figure 2d, Supplementary Table 3 and Supplementary Figure S5). sL359V showed binding efficiency similar to WT, Class II mutants had 1.7- to 5.7-fold reduction, gT355del had 11-fold reduction in affinity, whereas gR361L and gC373R (Emberger syndrome mutants) were below the limits of detection. We also included gR396Q in this assay as in one report, a Japanese girl with gR396Q, in addition to MDS and ID, developed lymphedema. ${ }^{30}$ This mutant showed 13 -fold reduction in affinity. To date, neither Class I nor || mutants have been associated with lymphedema and we predict individuals carrying Class III mutants (gT355del, gR361L, gC373R and gR396Q) to be predisposed to lymphedema.

Our previous work indicated that $\mathrm{gC} 373 \mathrm{R}$ is poorly folded due to mutation of a zinc-coordinating residue and gT354M is partially unfolded $^{34}$ (Figure 2a). Homology modeling indicated that mutation of positively charged arginine residues $(\mathrm{gR} 361 \mathrm{~L}$, sR362Q, gR396Q and gR398W) probably disrupts critical interactions with DNA without affecting ZF2 structure (Supplementary Figure S6). To test the effect of mutation on ZF2, far-UV circular dichroism spectropolarimetry and one-dimensional proton nuclear magnetic resonance spectroscopy were used to assess secondary and tertiary structure, respectively. gT355del was shown to be partially unfolded, similar to gT354M, with high levels of secondary structure, but moderate disruptions of tertiary structure compared with WT. The arginine mutants have WT secondary and tertiary structure (Supplementary Figure S7). There was no correlation between the mechanism of disruption and the magnitude of DNA-binding reduction, suggesting that functional 

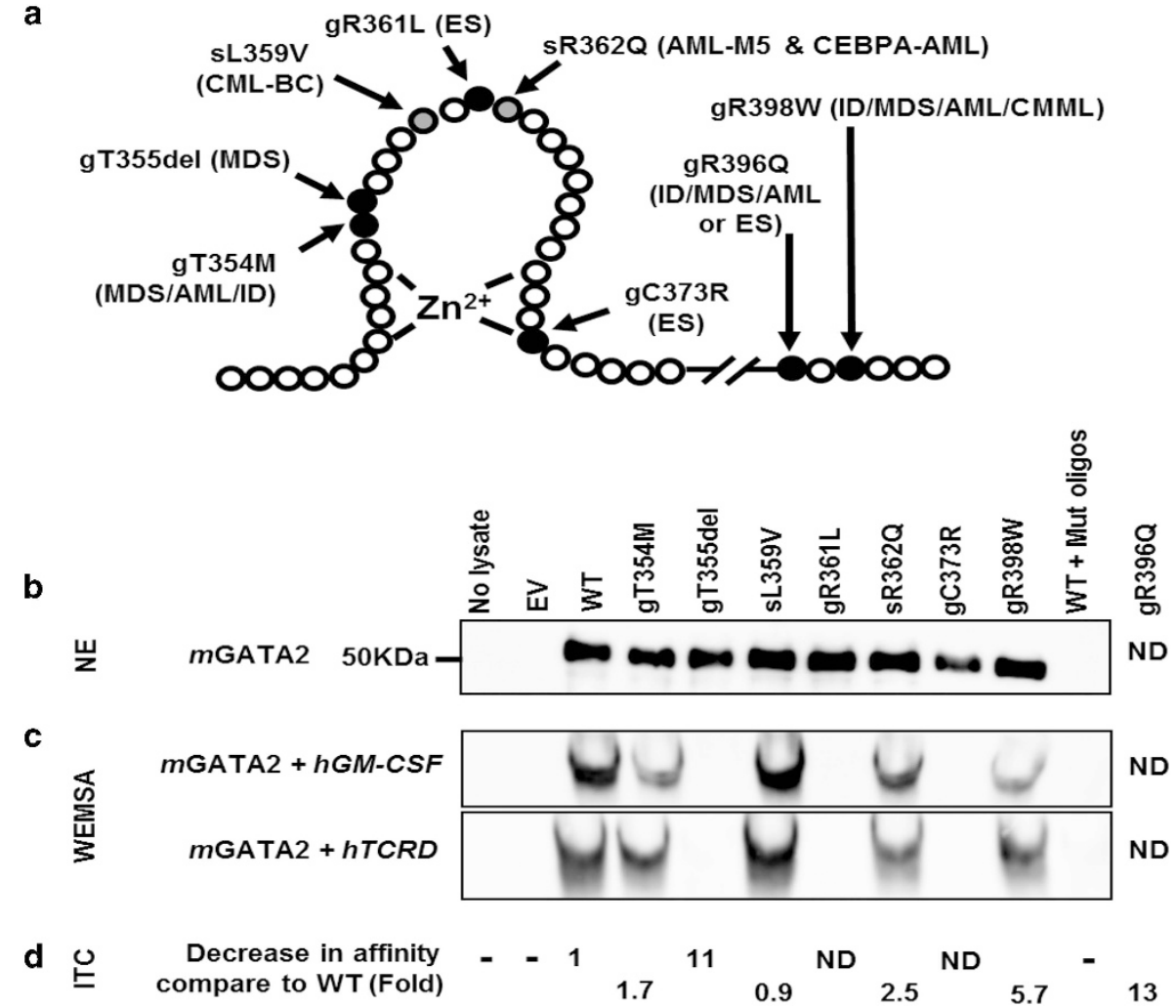

Figure 2. Mutations in ZF2 domain perturb GATA2 activity and function. (a) Location of missense mutations in GATA2 ZF2 domain investigated in this study and their associated phenotypes. (b) GATA2 mutants differ in their DNA-binding affinity. Expression of full-length mGATA2 proteins (nuclear extracts (NE); $10 \mu \mathrm{g}$ each) detected by western blotting with an anti-GATA2 antibody. (c) GATA2 NEs (10 $\mu \mathrm{g}$ ) were bound to hGM-CSF and $h T C R D$ enhancer oligonucleotide probes and western blotting electromobility shift assays were performed using anti-GATA2 antibody. Oligonucleotide probes with mutated GATA-binding site (Mut) for hGM-CSF or hTCRD enhancer were used as negative controls. ND, not determined. (d) DNA-binding affinity of $h$ GATA2 ZF2 domain quantified by isothermal titration calorimetry.

assays are required to determine DNA-binding affinity. Collectively, alterations to the integrity of ZF2 structure and mutation of key DNA-binding residues can independently and variably disrupt GATA2 DNA binding.

GATA2 mutants display differentially altered transactivation activity and protein-protein interactions

To determine the effect of GATA2 mutations on the ability to transactivate transcription, we performed luciferase reporter assays on known GATA2-responsive promoters ( $L Y L 1$ and CSF1R) and enhancer (CD34). All mutants displayed a reduced ability to transactivate transcription from at least one of these GATA2responsive elements relative to WT GATA2, except for SL359V (Figure 3 and Supplementary Figures S8a and b), which we and others have shown displays WT or mild gain-of-function (GOF) activity. ${ }^{4,20}$ Class III mutants with least DNA-binding affinity (gT355del, gR361L and gC373R; Figure 3) showed the most marked reduction in transactivation, including complete abrogation of transactivation in some instances, whereas Class II mutants (gT354M, sR362Q, gR398W and sL321F (Supplementary Figure $\mathrm{S} 8 \mathrm{C}$ )) displayed residual transactivation capacity.

Previously, we have shown that GATA2 and PU.1 (SPI1) synergistically upregulate CSF1R promoter activity. ${ }^{4}$ Here, all GATA2 mutants (except for SL359V) showed reduced synergistic transactivation with PU.1 (Figure 3c); however, all mutants retained some ability to activate transcription with PU.1 relative to PU.1 alone (Supplementary Figure S8d). Even mutants with complete loss of DNA binding were able to co-activate with PU.1. Consistent with this, in co-immunoprecipitation assays, all GATA2 mutants maintained an ability to interact with PU.1 (Figure 4 and
Supplementary Figure S9), which has been shown to occur via ZF2. ${ }^{38}$ Strikingly, gT354M and gC373R showed enhanced affinity for PU.1. Together these data show that although transactivation via GATA2-responsive elements generally correlates with DNAbinding activity, interaction with and co-activation with PU.1 differs between mutants in a non-DNA-binding-dependent manner.

GATA2 mutants perturb differentiation of hematopoietic stem and progenitor cells

We next investigated the effects of GATA2 mutations on hematopoiesis using colony-forming assays. mGATA2 WT and mutants were retrovirally transduced into murine LSK cells using pMSCV-mGata2-IRES-GFP and sorted for green fluorescent protein (GFP) positivity. Similar levels of mGATA2 WT and mutants in LSK cells were achieved and confirmed using quantitative reverse transcriptase-PCR (Supplementary Figure S10). As shown previously, ${ }^{39}$ WT GATA2 was able to efficiently suppress colony formation and multilineage differentiation of progenitor cells, with the exception of the erythroid lineage (colony forming uniterythroid, BFU-E) (Figure 5). All GATA2 mutants displayed significant de-repression of colony formation, restoring colony numbers to similar to empty vector (EV) (Figure 5a). Interestingly, this included $S L 359 \mathrm{~V}$, indicating that de-repression of colonyforming ability is, at least in some cases, uncoupled from effects on DNA binding and transactivation ability. Granulocyte colonyforming units mirrored total colony numbers (Figure $5 \mathrm{~b}$ ). A similar increasing trend was also observed in colony formation for granulocyte-macrophage/granulocyte, erythrocyte, monocyte and megakaryocyte progenitors (Figure $5 \mathrm{c}$ ). In contrast to 
a

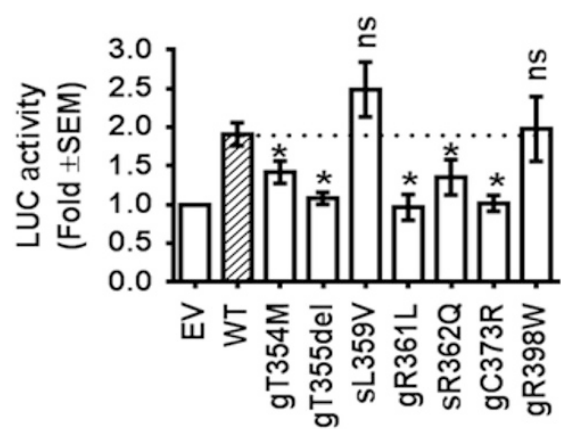

b

LYL1 promoter

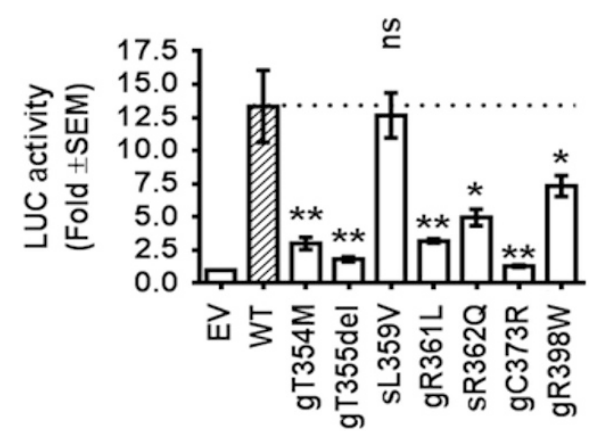

C CSF1R promoter

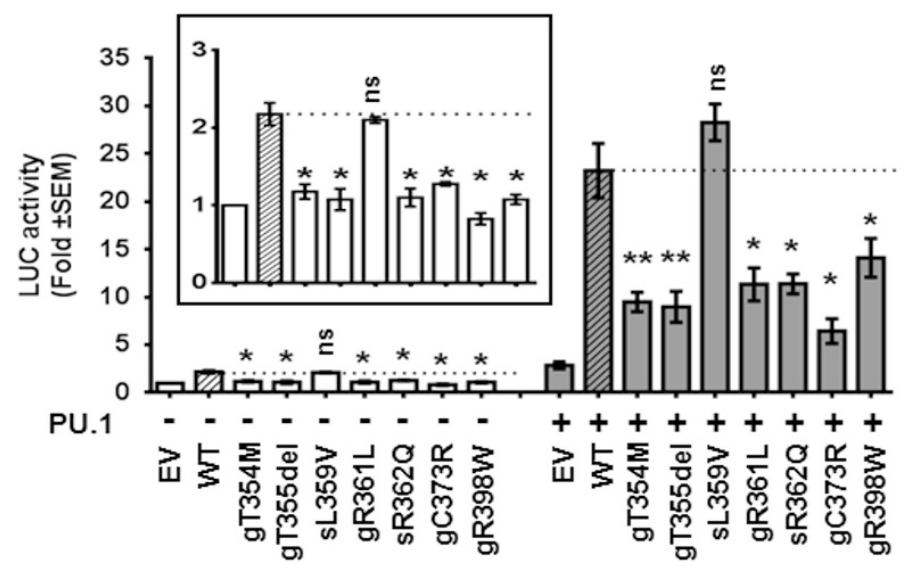

Figure 3. GATA2 mutants display variable loss of transactivation capacity on known GATA2-responsive elements. (a) HEK293 cells were cotransfected with GATA2-responsive CD34 enhancer-LUC reporter and GATA2 (WT or mutants) expression constructs or EV. (b) Cos-7 cells were co-transfected with LYL1 promoter-LUC reporter and GATA2 expression constructs. (c) GATA2 expression constructs were co-transfected with CSF1R promoter-LUC into Cos-7 cells (white columns). Inset, a magnified view of the first nine conditions. The synergistic effect of GATA2 with PU.1 on the CSF1R promoter was investigated by co-transfecting GATA2 expression constructs with PU.1 (grey columns). Results are fold change compared with EV ( \pm s.e.m., four independent biological replicates). Differences between WT (hatch) and mutants were calculated. ns: not significant, ${ }^{*} P<0.05$ and ${ }^{* *} P<0.01$.
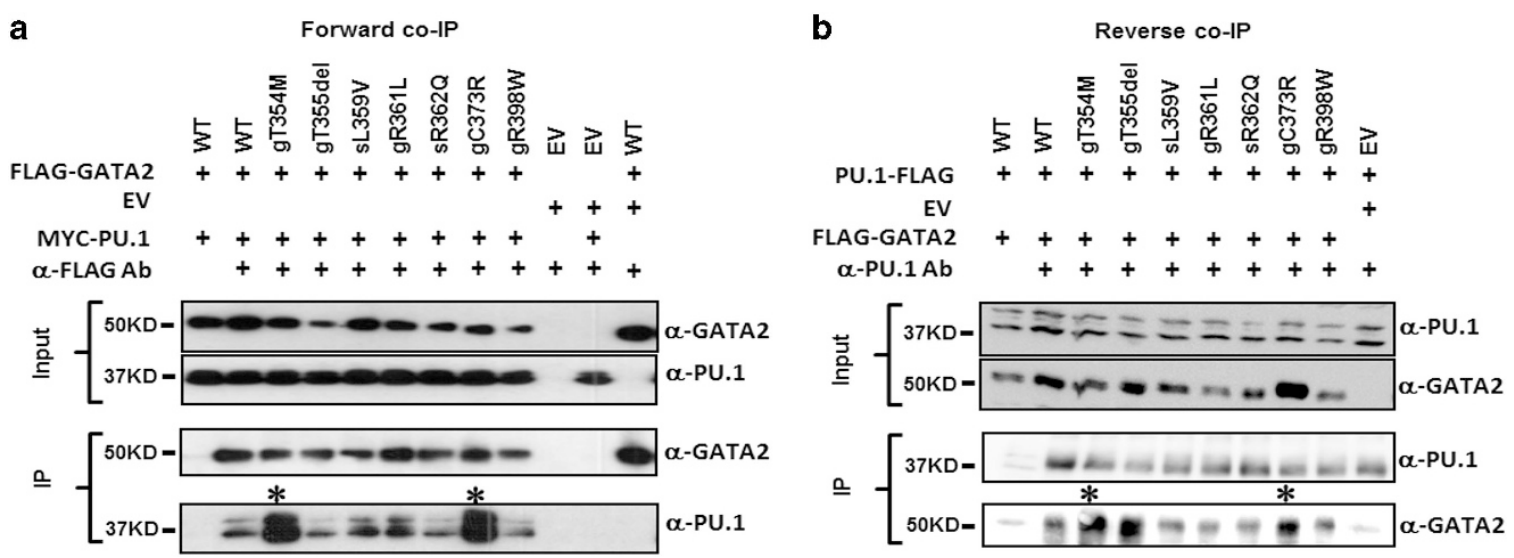

Figure 4. GATA2 mutants alter interaction with PU.1. (a) PU.1 was co-immunoprecipitated (co-IP) with GATA2 using anti-FLAG antibody (forward). (b) GATA2 was co-IP with PU.1 using anti-PU.1 antibody (reverse). Western blot analyses were performed with anti-GATA2 or antiPU.1 antibody. gT354M and gC373R (*) show increased binding activity to PU.1.

granulocyte CFU formation, not all GATA2 mutants de-repressed macrophage CFUs, with Class I and II mutants with the most preserved DNA-binding activity (sL359V, gT354M and sR362Q) maintaining WT levels of macrophage CFU repression (Figure $5 \mathrm{~d}$ ). Notably, the two somatic mutations also promoted erythroid differentiation (Figure 5e). This suggests that there are both
DNA-dependent and independent effects of GATA2 mutations on myeloid lineage differentiation, with mutations associated most frequently with development of acute leukemic states (gT354M with familial AML, sL359V with chronic myeloid leukemia in blast crisis and SR362Q with sporadic AML) maintaining an ability to repress macrophage progenitor differentiation. 


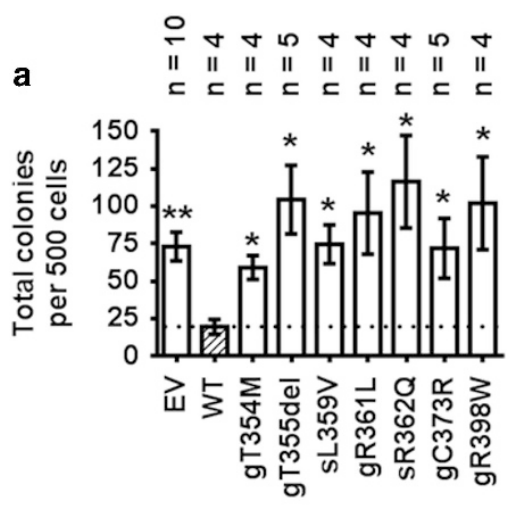

b
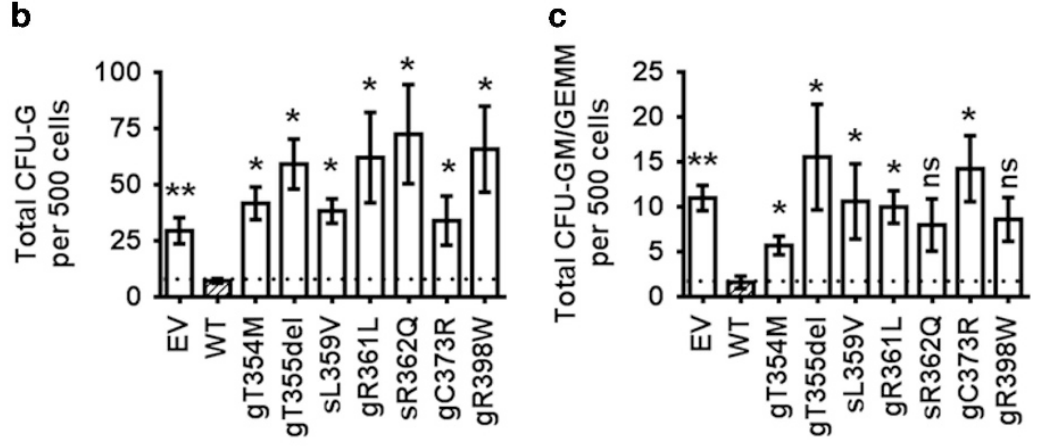

d

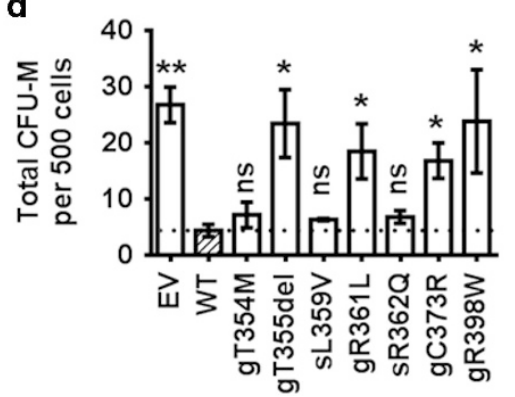

e

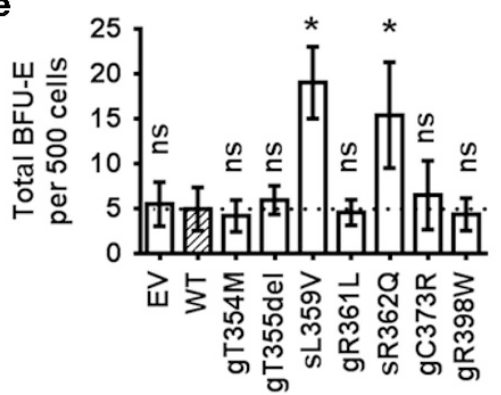

Figure 5. GATA2 mutants differentially impact clonogenic expansion and hematopoietic differentiation. (a) Enumeration of absolute colony numbers. GATA2 WT (hatch) was used as a comparator for all analyses. (b-e) Colonies were typed by morphology and numbers were reported. (b) Granulocyte CFU (CFU-G), (c) colony formation for granulocyte-macrophage/granulocyte, erythrocyte, monocyte and megakaryocyte progenitors (CFU-GM/GEMM), (d) macrophage CFU (CFU-M) and (e) BFU-E. All results are plotted as mean \pm s.e.m. with $n$ independent biological replicates. ns: not significant, ${ }^{*} P<0.05$ and ${ }^{* *} P<0.01$.

To examine in vivo effects of GATA2 mutants on hematopoiesis, we transplanted transduced LSK cells (EV, GATA2 WT, gT354M or g(373R), along with untransduced helper marrow, into lethally irradiated recipient mice and monitored the percentage of GFPpositive cells in peripheral blood as a measure of contribution to engraftment (Supplementary Table 4 and Supplementary Figure S11). GFP-positive cells were observed in the peripheral blood of all mice transduced with EV at time points up to 20 weeks post transplantation $(n=8 / 8)$. Consistent with the ability of increased GATA2 expression to block hematopoiesis in normal mice and to impair progenitor growth (above), only $40 \%$ of mice transplanted with WT GATA2 LSK had GFP-positive cells (4/10) and no GFP-positive cells were seen at time points of 10 weeks or later. Similar numbers of mice with GFP contribution to engraftment were observed for both GATA2 mutants (T354M $n=4 / 9$, C373R $n=5 / 7)$, although in each case at least 2 mice maintained GFPpositive cells at 14 weeks, indicating potential loss of inhibitory activity of WT GATA2 in this context. Mice were aged for up to 12 months and during this time no differences in survival or development of hematological disorders were observed.

\section{DISCUSSION}

Germline GATA2 mutations underlie the development of hematological, immunological and lymphatic disorders. Diverse phenotypes in patients harboring different germline GATA2 mutations have led us to investigate the effect of the three most prevalent GATA2 missense mutations (gT354M, gR396Q and gR398W). These mutants appear to impact on disease subtypes (for example, MDS/ AML and chronic myelomonocytic leukemia) and exhibit differences in age of onset. Recently, Ding et al. ${ }^{40}$ reported two cases of gR396Q mutation in patients who developed MDS at younger age, consistent with our data that it predisposes predominantly to MDS and with early onset. These data support the concept of genetic 'predestination' where initiator or founder mutations in a protein or even domain confer increased tendency to direct or constrain the impact of subsequent mutations and phenotypes. ${ }^{41,42}$

Recurrent and clinically important GATA2 mutations in ZF2 perturb protein folding and/or DNA-binding affinity. Homology modeling predicts that the four arginine residues (R361, R362, R396 and R398) and L359 ${ }^{4}$ directly contact DNA, whereas T354, T355 and C373 are more likely involved in maintaining ZF2 structural integrity. We have proposed three classes of GATA2 mutations based on DNA-binding affinity (Figure 6); enhanced, reduced and greatly reduced. Interestingly, mutations within each class lead to different clinical outcomes. The Class I mutant is a unique case of somatic mutation associated with driving chronic myeloid leukemia into blast crisis $^{20}$ and has not been seen in MDS/AML. Class II mutants are associated with myeloid malignancies and/or ID. In addition to Class II phenotypes, Class III mutants also predispose to primary lymphedema (we predict this for gT355del) consistent with complete loss-of-function (LOF) of one GATA2 allele being required for predisposition to primary lymphedema, as proposed by us and others., 80,19 This is further supported by our findings where heterozygous conditional knockout of Gata2 results in defective lymphatic vessel structure and vascular transport in adult mice. ${ }^{34}$ Together, these data support what is seen clinically, where germline GATA2 mutants with a threshold of residual activity predispose to myeloid malignancy and/or ID, but not to lymphedema.

Our results together with the published data suggest that DNAbinding affinity alone cannot explain the association between specific GATA2 ZF2 mutations and hematological malignancy subtypes. We show for the first time that ZF2 mutations can drastically affect its ability to interact with PU.1, a known key myeloid transcription factor-binding partner. Specifically, gT354M and gC373R with altered ZF2 tertiary structure bind PU.1 more tightly. PU.1 is an E-twenty-six (ETS) transcription factor that 


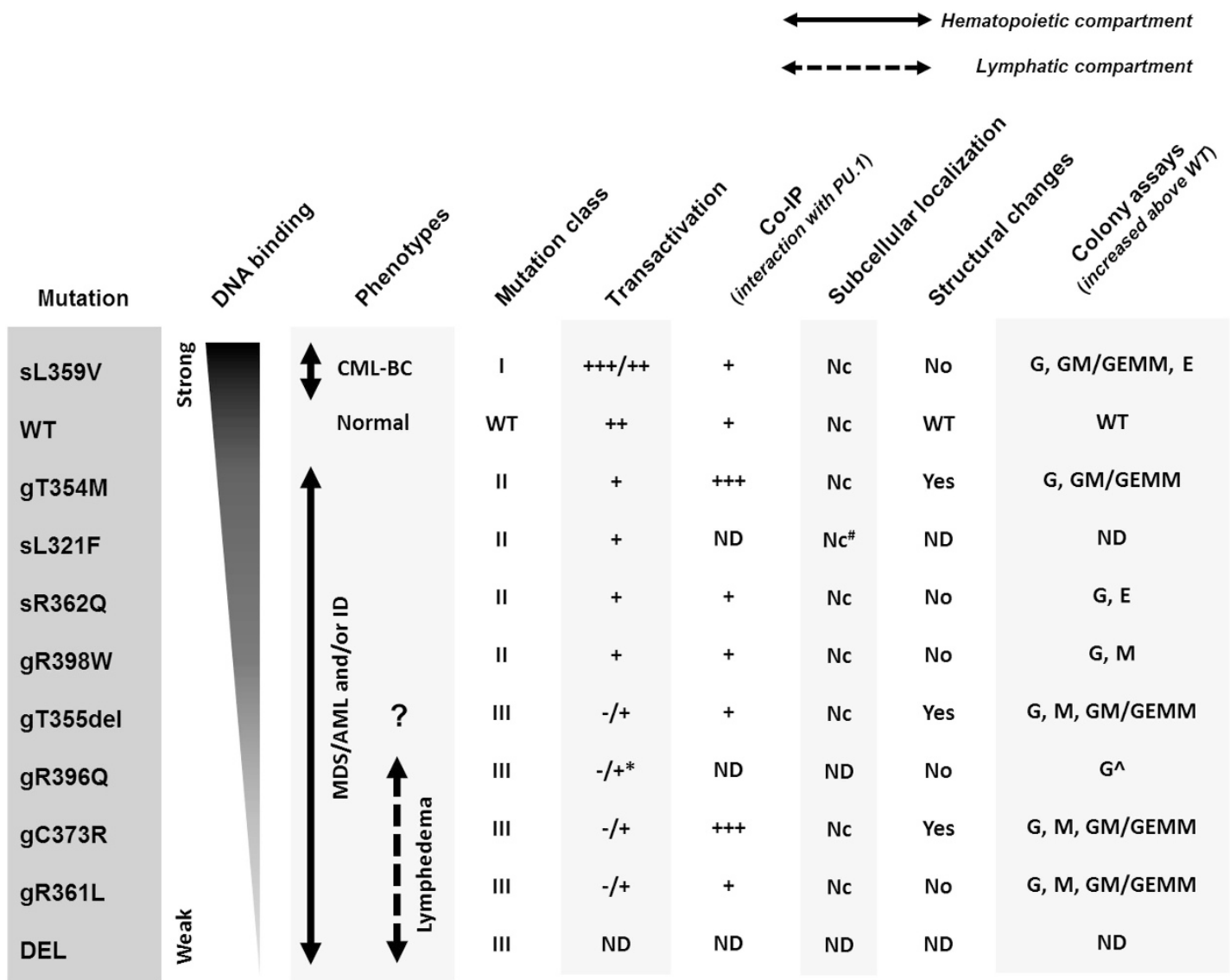

Figure 6. Summary of functional assays for GATA2 mutant allelic series in this study. GATA2 mutants are listed in descending order of DNAbinding affinity. Results of transactivation, co-IP, subcellular localization, structural changes and colony assays are summarized. DEL indicates partial or whole gene deletion and mutants with premature termination; Nc, nuclear; ND, not determined. \#See Greif et al. ${ }^{24}{ }^{*}$ See Kazenwadel et al. ${ }^{34} \wedge$ See Cortes-Lavaud et al. ${ }^{47}$

induces expression of genes pivotal to myeloid differentiation. ${ }^{43}$ Although mutations in PU.1 gene and its regulatory region are relatively rare in cytogenetically normal MDS and $A M L,{ }^{44,45}$ studies have shown that PU.1 activity is compromised in many AML cases. For instance, fusion protein pro-myelocytic leukemia ZF/retinoic acid receptor alpha (RARA) directly interacts with PU.1 resulting in sequestration of PU.1. ${ }^{46}$ Similarly, enhanced binding by these GATA2 mutants may sequester both GATA2 and PU.1 from their normal roles in myelopoiesis. How ZF2 mutations affect the binding to other GATA2-binding partners such as CEBPA remains to be determined. We hypothesize that some GATA2 mutants may impair hematopoiesis via aberrant sequestration of key hematopoietic transcription factors resulting in disruption of normal cellular processes including differentiation, proliferation and survival. Further, gT354M and gR396Q were reported to attenuate chromatin occupancy, reduce endogenous target gene activation and disrupt endogenous GATA2 WT binding suggestive of dominant-negative properties. ${ }^{47,48}$ Moreover, our colonyformation assays are consistent with those reported previously for gR396Q. ${ }^{47}$ Hence, the extent of loss of DNA binding and transactivation, and changes to protein-protein interactions may contribute to the clinically diverse outcomes of GATA2-related disease.

GATA2 WT or mutants have varying effects on growth and differentiation of hematopoietic progenitors. Expression of GATA2 WT results in a profound block in clonogenic growth, colony numbers and differentiation in vitro. This is consistent with elevated GATA2 levels in hematopoietic stem cells driving quiescence $^{49,50}$ and the role of GATA2 in maintaining the 'stemness' of these cells. ${ }^{51,52}$ Expression of GATA2 is known to perturb normal hematopoiesis and cause pancytopenia in transplanted mice. ${ }^{39}$ Here, all GATA2 mutants, independent of DNA-binding affinity, displayed loss of progenitor growth inhibition and we speculate that under certain conditions GATA2 mutants are unable to restrain growth/cycling, contributing to stem cell pool depletion and exhaustion. This may be particularly important on a background of persistent infection and/or hematopoietic stress such as in bone marrow failure, as it has been proposed previously for MonoMAC/DCML deficiency syndromes. ${ }^{11-14}$ Colony-formation assays show an increase in granulocytic progenitors following expression of GATA2 mutants consistent with skewed differentiation causing the monocytopenia observed in ID syndrome. Although it is not clear why monocytes are preferentially depleted, GATA2 mutated patients have better preservation of neutrophils (granulocytes) compared with GATA2 WT MDS or idiopathic aplastic anemia patients. ${ }^{17,19}$ Conversely, erythroid progenitors are selectively resistant to the suppressive effects of sL359V and sR362Q allowing for colony expansion. Recently, Ping et al..$^{53}$ discovered a subset of acute erythroid leukemia patients with somatic GATA2 mutations in ZF1 domain. Although there is no clinical evidence directly linking sL359V and sR362Qs to erythroid leukemia, these GATA2 ZF2 mutants resemble GATA2 ZF1 mutations, in that they commonly arise in a background of pre-existing mutations (for example, CEBPA and $B C R-A B L$ ). In contrast to the predominance of GATA2 germline mutations in ZF2, most somatic GATA2 mutations cluster within ZF1. GATA2 ZF1 (for example, sL321F) mutations and a sole ZF2 (sR362Q) mutation are often associated with AML harboring 
biallelic CEBPA mutations (biCEBPA AML; 25\% contain ZF1 or sR362Q mutations). ${ }^{22-24,36,37}$ This association appears to be less for germline ZF2 mutations, which seem rarely to co-occur with CEBPA mutations implying different leukemogenic mechanisms for ZF1 and ZF2 mutations. Acquired initiator mutations in CEBPA may generate permissive pre-leukemic environments enabling selective forces for ZF1 or particular ZF2 (R362) mutations. Various substitutions of $\mathrm{R} 362$ (sR362Q/P/G) have been reported in cytogenetically normal pediatric $\mathrm{AML}^{36,37}$ and adult biCEBPA $\mathrm{AML}^{22,23}$ highlighting the importance of changes to this DNAbinding residue in sporadic leukemia development.

Our functional studies on clinically important GATA2 ZF2 mutants provide new insights into mechanisms interacting with environmental stresses on the hematopoietic and lymphatic compartments that contribute to myeloid malignancies, ID with a unique spectrum of permissive infections and/or lymphedema. We demonstrate the complexity of amino acid mutation specific phenotypes important in sporadic AML subtypes in addition to hereditary MDS/AML subtypes. Further mechanistic studies are warranted to better understand the complex interplay of genetic and environment causal factors in attempt to treat or prevent development of these disorders.

\section{CONFLICT OF INTEREST}

The authors declare no conflict of interest.

\section{ACKNOWLEDGEMENTS}

This work was supported by funding from National Health and Medical Research Council, (APP1002317 and APP1023059), Cancer Council of South Australia (APP626953) and Adelaide Scholarships International from the University of Adelaide, Australia.

\section{AUTHOR CONTRIBUTIONS}

C-EC, ALB, CNH and HSS wrote the manuscript. CNH, ALB, MK-H, JMM, RJD and HSS planned experiments. C-EC, PV, PHS, YKL, PJB, MB and GAE performed functional experiments. SWL provided expert advice on ex vivo experimentation and DTOY on clinical aspects of data analysis. All authors critically reviewed and approved the manuscript.

\section{REFERENCES}

1 Arber DA, Orazi A, Hasserjian R, Thiele J, Borowitz MJ, Le Beau MM et al. The 2016 revision to the World Health Organization classification of myeloid neoplasms and acute leukemia. Blood 2016; 127: 2391-2405.

2 Song WJ, Sullivan MG, Legare RD, Hutchings S, Tan X, Kufrin D et al. Haploinsufficiency of CBFA2 causes familial thrombocytopenia with propensity to develop acute myelogenous leukaemia. Nat Genet 1999; 23: 166-175.

3 Smith ML, Cavenagh JD, Lister TA, Fitzgibbon J. Mutation of CEBPA in familial acute myeloid leukemia. N Engl J Med 2004; 351: 2403-2407.

4 Hahn CN, Chong CE, Carmichael CL, Wilkins EJ, Brautigan PJ, Li XC et al. Heritable GATA2 mutations associated with familial myelodysplastic syndrome and acute myeloid leukemia. Nat Genet 2011; 43: 1012-1017.

5 Marquez R, Hantel A, Lorenz R, Neistadt B, Wong J, Churpek JE et al. A new family with a germline ANKRD26 mutation and predisposition to myeloid malignancies. Leuk Lymph 2014; 55: 2945-2946.

6 Lewinsohn M, Brown AL, Weinel LM, Phung C, Rafidi G, Lee MK et al. Novel germ line DDX41 mutations define families with a lower age of MDS/AML onset and lymphoid malignancies. Blood 2016; 127: 1017-1023.

7 Wlodarski MW, Hirabayashi S, Pastor V, Stary J, Hasle H, Masetti R et al. Prevalence, clinical characteristics, and prognosis of GATA2-related myelodysplastic syndromes in children and adolescents. Blood 2016; 127: 1387-1397.

8 Kazenwadel J, Secker GA, Liu YJ, Rosenfeld JA, Wildin RS, Cuellar-Rodriguez J et al. Loss-of-function germline GATA2 mutations in patients with MDS/AML or MonoMAC syndrome and primary lymphedema reveal a key role for GATA2 in the lymphatic vasculature. Blood 2012; 119: 1283-1291.

9 Mansour S, Connell F, Steward C, Ostergaard P, Brice G, Smithson S et al. Emberger syndrome-primary lymphedema with myelodysplasia: report of seven new cases. Am J Med Genet A 2010; 152A: 2287-2296.
10 Ostergaard P, Simpson MA, Connell FC, Steward CG, Brice G, Woollard WJ et al. Mutations in GATA2 cause primary lymphedema associated with a predisposition to acute myeloid leukemia (Emberger syndrome). Nat Genet 2011; 43: 929-931.

11 Hsu AP, Sampaio EP, Khan J, Calvo KR, Lemieux JE, Patel SY et al. Mutations in GATA2 are associated with the autosomal dominant and sporadic monocytopenia and mycobacterial infection (MonoMAC) syndrome. Blood 2011; 118: 2653-2655.

12 Vinh DC, Patel SY, Uzel G, Anderson VL, Freeman AF, Olivier KN et al. Autosomal dominant and sporadic monocytopenia with susceptibility to mycobacteria, fungi, papillomaviruses, and myelodysplasia. Blood 2010; 115: 1519-1529.

13 Bigley V, Haniffa M, Doulatov S, Wang XN, Dickinson R, McGovern N et al. The human syndrome of dendritic cell, monocyte, B and NK lymphoid deficiency. $J$ Exp Med 2011; 208: 227-234.

14 Dickinson RE, Griffin H, Bigley V, Reynard LN, Hussain R, Haniffa M et al. Exome sequencing identifies GATA-2 mutation as the cause of dendritic cell, monocyte, $B$ and NK lymphoid deficiency. Blood 2011; 118: 2656-2658.

15 Pasquet M, Bellanne-Chantelot C, Tavitian S, Prade N, Beaupain B, Larochelle O et al. High frequency of GATA2 mutations in patients with mild chronic neutropenia evolving to MonoMac syndrome, myelodysplasia, and acute myeloid leukemia. Blood 2013; 121: 822-829.

16 Mace EM, Hsu AP, Monaco-Shawver L, Makedonas G, Rosen JB, Dropulic L et al. Mutations in GATA2 cause human NK cell deficiency with specific loss of the CD56 (bright) subset. Blood 2013; 121: 2669-2677.

17 Ganapathi KA, Townsley DM, Hsu AP, Arthur DC, Zerbe CS, Cuellar-Rodriguez J et al. GATA2 deficiency-associated bone marrow disorder differs from idiopathic aplastic anemia. Blood 2015; 125: 56-70.

18 Townsley DM, Hsu A, Dumitriu B, Holland SM, Young NS. Regulatory Mutations in GATA2 Associated with Aplastic Anemia. Blood 2012; 120: 3448. (ASH Annual Meeting Abstracts).

19 Dickinson RE, Milne P, Jardine L, Zandi S, Swierczek SI, McGovern N et al. The evolution of cellular deficiency in GATA2 mutation. Blood 2014; 123: 863-874.

20 Zhang SJ, Ma LY, Huang QH, Li G, Gu BW, Gao XD et al. Gain-of-function mutation of GATA-2 in acute myeloid transformation of chronic myeloid leukemia. Proc Natl Acad Sci USA 2008; 105: 2076-2081.

21 Yan XJ, Xu J, Gu ZH, Pan CM, Lu G, Shen Y et al. Exome sequencing identifies somatic mutations of DNA methyltransferase gene DNMT3A in acute monocytic leukemia. Nat Genet 2011; 43: 309-315.

22 Fasan A, Eder C, Haferlach C, Grossmann V, Kohlmann A, Dicker F et al. GATA2 mutations are frequent in intermediate-risk karyotype AML with biallelic CEBPA mutations and are associated with favorable prognosis. Leukemia 2013; 27: 482-485.

23 Green CL, Tawana K, Hills RK, Bodor C, Fitzgibbon J, Inglott S et al. GATA2 mutations in sporadic and familial acute myeloid leukaemia patients with CEBPA mutations. Br J Haematol 2013; 161: 701-705.

24 Greif PA, Dufour A, Konstandin NP, Ksienzyk B, Zellmeier E, Tizazu B et al. GATA2 zinc finger 1 mutations associated with biallelic CEBPA mutations define a unique genetic entity of acute myeloid leukemia. Blood 2012; 120: 395-403.

25 Marceau-Renaut A, Guihard S, Castaigne S, Dombret H, Preudhomme C, Cheok M. Classification of CEBPA mutated acute myeloid leukemia by GATA2 mutations. Am J Hematol 2015; 90: E93-E94.

26 Bodor C, Renneville A, Smith M, Charazac A, lqbal S, Etancelin P et al. Germ-line GATA2 p.THR354MET mutation in familial myelodysplastic syndrome with acquired monosomy 7 and ASXL1 mutation demonstrating rapid onset and poor survival. Haematologica 2012; 97: 890-894.

27 Cuellar-Rodriguez J, Gea-Banacloche J, Freeman AF, Hsu AP, Zerbe CS, Calvo KR et al. Successful allogeneic hematopoietic stem cell transplantation for GATA2 deficiency. Blood 2011; 118: 3715-3720.

28 Holme H, Hossain U, Kirwan M, Walne A, Vulliamy T, Dokal I. Marked genetic heterogeneity in familial myelodysplasia/acute myeloid leukaemia. $\mathrm{Br} J$ Haematol 2012; 158: 242-248.

29 Hsu AP, Johnson KD, Falcone EL, Sanalkumar R, Sanchez L, Hickstein DD et al. GATA2 haploinsufficiency caused by mutations in a conserved intronic element leads to MonoMAC syndrome. Blood 2013; 121: 3830-3837, S3831-3837.

30 Ishida H, Imai K, Honma K, Tamura S, Imamura T, Ito M et al. GATA-2 anomaly and clinical phenotype of a sporadic case of lymphedema, dendritic cell, monocyte, B- and NK-cell (DCML) deficiency, and myelodysplasia. Eur J Pediatr 2012; 171: 1273-1276.

31 Kaur J, Catovsky D, Valdimarsson $\mathrm{H}$, Jensson O, Spiers AS. Familial acute myeloid leukaemia with acquired Pelger-Huet anomaly and aneuploidy of $\mathrm{C}$ group. Br Med J 1972; 4: 327-331.

32 Spinner MA, Sanchez LA, Hsu AP, Shaw PA, Zerbe CS, Calvo KR et al. GATA2 deficiency: a protean disorder of hematopoiesis, lymphatics, and immunity. Blood 2014; 123: 809-821.

33 West RR, Hsu AP, Holland SM, Cuellar-Rodriguez J, Hickstein DD. Acquired ASXL1 mutations are common in patients with inherited GATA2 mutations and correlate with myeloid transformation. Haematologica 2014; 99: 276-281. 
34 Kazenwadel J, Betterman KL, Chong CE, Stokes PH, Lee YK, Secker GA et al. GATA2 is required for lymphatic vessel valve development and maintenance. J Clin Invest 2015; 125: 2979-2994.

35 Fine JP, Gray RJ. A proportional hazards model for the subdistribution of a competing risk. J Am Stat Assoc 1999; 94: 496-509.

36 Luesink M, Hollink IH, van der Velden VH, Knops RH, Boezeman JB, de Haas V et al. High GATA2 expression is a poor prognostic marker in pediatric acute myeloid leukemia. Blood 2012; 120: 2064-2075.

37 Shiba N, Funato M, Ohki K, Park MJ, Mizushima Y, Adachi S et al. Mutations of the GATA2 and CEBPA genes in paediatric acute myeloid leukaemia. $\mathrm{Br} J$ Haematol 2014; 164: 142-145.

38 Zhang P, Behre G, Pan J, Iwama A, Wara-Aswapati N, Radomska HS et al. Negative cross-talk between hematopoietic regulators: GATA proteins repress PU.1. Proc Natl Acad Sci USA 1999; 96: 8705-8710.

39 Persons DA, Allay JA, Allay ER, Ashmun RA, Orlic D, Jane SM et al. Enforced expression of the GATA-2 transcription factor blocks normal hematopoiesis. Blood 1999; 93: 488-499.

40 Ding LW, Ikezoe T, Tan KT, Mori M, Mayakonda A, Chien W et al. Mutational profiling of a MonoMAC syndrome family with GATA2 deficiency. Leukemia 2017; 31: 244-245.

41 Cazzola M, Della Porta MG, Malcovati L. The genetic basis of myelodysplasia and its clinical relevance. Blood 2013; 122: 4021-4034.

42 Papaemmanuil E, Gerstung M, Malcovati L, Tauro S, Gundem G, Van Loo P et al. Clinical and biological implications of driver mutations in myelodysplastic syndromes. Blood 2013; 122: 3616-3627, quiz 3699.

43 Nerlov C, Graf T. PU.1 induces myeloid lineage commitment in multipotent hematopoietic progenitors. Genes Dev 1998; 12: 2403-2412.

44 Cheng JX, Anastasi J, Watanabe K, Kleinbrink EL, Grimley E, Knibbs R et al. Genome-wide profiling reveals epigenetic inactivation of the PU.1 pathway by histone $\mathrm{H} 3$ lysine 27 trimethylation in cytogenetically normal myelodysplastic syndrome. Leukemia 2013; 27: 1291-1300.

45 Mueller BU, Pabst T, Osato M, Asou N, Johansen LM, Minden MD et al. Heterozygous PU.1 mutations are associated with acute myeloid leukemia. Blood 2002; 100: $998-1007$.
46 Seshire A, Rossiger T, Frech M, Beez S, Hagemeyer H, Puccetti E. Direct interaction of PU.1 with oncogenic transcription factors reduces its serine phosphorylation and promoter binding. Leukemia 2012; 26: 1338-1347.

47 Cortes-Lavaud X, Landecho MF, Maicas M, Urquiza L, Merino J, Moreno-Miralles I et al. GATA2 germline mutations impair GATA2 transcription, causing haploinsufficiency: functional analysis of the p.Arg396Gln mutation. J Immunol 2015; 194: 2190-2198.

48 Katsumura KR, Yang C, Boyer ME, Li L, Bresnick EH. Molecular basis of crosstalk between oncogenic Ras and the master regulator of hematopoiesis GATA-2. EMBO Rep 2014; 15: 938-947.

49 Venezia TA, Merchant AA, Ramos CA, Whitehouse NL, Young AS, Shaw CA et al. Molecular signatures of proliferation and quiescence in hematopoietic stem cells. PLoS Biol 2004; 2: e301.

50 Tipping AJ, Pina C, Castor A, Hong D, Rodrigues NP, Lazzari L et al. High GATA-2 expression inhibits human hematopoietic stem and progenitor cell function by effects on cell cycle. Blood 2009; 113: 2661-2672.

51 Ramalho-Santos M, Yoon S, Matsuzaki Y, Mulligan RC, Melton DA. 'Stemness': transcriptional profiling of embryonic and adult stem cells. Science 2002; 298: 597-600.

52 Snow JW, Trowbridge JJ, Johnson KD, Fujiwara T, Emambokus NE, Grass JA et al. Context-dependent function of 'GATA switch' sites in vivo. Blood 2011; 117: 4769-4772.

53 Ping $N$, Sun $A$, Song $Y$, Wang $Q$, Yin J, Cheng W et al. Exome sequencing identifies highly recurrent somatic GATA2 and CEBPA mutations in acute erythroid leukemia. Leukemia 2017; 31: 195-202.

(c) (1) $\odot$ This work is licensed under a Creative Commons AttributionNonCommercial-NoDerivs 4.0 International License. The images or other third party material in this article are included in the article's Creative Commons license, unless indicated otherwise in the credit line; if the material is not included under the Creative Commons license, users will need to obtain permission from the license holder to reproduce the material. To view a copy of this license, visit http:// creativecommons.org/licenses/by-nc-nd/4.0/

(c) The Author(s) 2018

Supplementary Information accompanies this paper on the Leukemia website (http://www.nature.com/leu) 